\title{
Projection Micro Stereolithography (PuSL) to 3D Print a Micro-Optofluidic Device for Two-Phase Slug Flow Detection: a Comparison with a PDMS-Based Device Manufactured by Resin Casting
}

Lorena Saitta ( $\square$ lorena.saitta@phd.unict.it )

University of Catania https://orcid.org/0000-0002-1423-8779

Giovanni Celano

University of Catania

Gianluca Cicala

University of Catania

Maria Elena Fragalà

INSTM-UDR CT, Viale Andrea Doria 6

Giovanna Stella

University of Catania

Matteo Barcellona

University of Catania

Claudio Tosto

University of Catania

Maide Bucolo

University of Catania

\section{Research Article}

Keywords: 3D printing, Projection Micro-Stereolithography, Micro-opticts, Microfluidics, Real-time monitoring, Two-phase flow.

Posted Date: December 8th, 2021

DOI: https://doi.org/10.21203/rs.3.rs-1107132/v1

License: (1) This work is licensed under a Creative Commons Attribution 4.0 International License.

Read Full License 


\section{Projection Micro Stereolithography (P $\mu \mathrm{SL}$ ) to 3D print a micro-optofluidic device for two-phase slug flow detection: a comparison with a PDMS-based device manufactured by resin casting}

Lorena Saitta $^{1 *}$, Giovanni Celano ${ }^{1}$, Gianluca Cicala ${ }^{1,2}$, Maria Elena Fragala

${ }^{2,3}$, Giovanna Stella ${ }^{4}$, Matteo Barcellona ${ }^{3}$, Claudio Tosto ${ }^{1}$ and Maide Bucolo ${ }^{4}$

${ }^{1}$ Department of Civil Engineering and Architecture, University of Catania, Viale Andrea Doria 6, Catania, 95125, Italy.

${ }^{2}$ INSTM-UDR CT, Viale Andrea Doria 6, Catania, 95125, Italy. ${ }^{3}$ Department of Chemical Sciences, University of Catania, Viale Andrea Doria 6, Catania, 95125, Italy.

${ }^{4}$ Department of Electrical Electronic and Computer Science Engineering, University of Catania, Viale Andrea Doria 6, Catania, 95125, Italy.

*Corresponding author(s). E-mail(s): lorena.saitta@phd.unict.it;

Contributing authors: giovanni.celano@unict.it; gianluca.cicala@unict.it; me.fragala@unict.it; giovanna.stella@phd.unict.it; matteo.barcellona@phd.unict.it; claudio.tosto@unict.it; maide.bucolo@unict.it;

\section{Abstract}

In this work, the use of Projection Micro Stereolithography (P $\mu \mathrm{SL})$ to $3 \mathrm{D}$ print a micro-optofluidic device for two-phase slug flow detection is presented. For comparison purposes a PDMS based device obtained by casting was also manufactured. The micro-optofluidic device has a microfluidic T-junction with a micro-optical section that consists 
of two optical fiber insertions used for two-phase slug flow detection. The working principle in the detection is based on a different light transmission correlated to the fluid interfering with the laser beam in a micro-channel section. The $3 \mathrm{D}$ printed material is fully characterized in terms of its surface properties and compared to PDMS used for standard construction using a master-slave casting procedure. The two devices were tested after the setup parameters for the detection were optimized using ANOVA for the 3D printed device. The comparisons of the two devices revealed that $3 \mathrm{D}$ printed device can be used for two-phase slug flow detection but future research is still need to obtain a $3 \mathrm{D}$ printed resin allowing to outperform PDMS.

Keywords: 3D printing, Projection Micro-Stereolithography, Micro-optics, Microfluidics, Real-time monitoring, Two-phase flow

\section{Introduction}

Lab-on-a-chip, also known as microfluidic devices, have gained a significant attention starting from 1990s [1]. In these devices a micrometric channel allows small volumes of fluids to be tested. Lab-on-a-chip devices can perform many operations usually need in conventional biochemistry laboratories, from sample preparation to reaction, detection, and other procedures [2]. Moreover, their use is wide spreading as they grant to get huge advantages in terms of low-cost in manufacturing, power consumption saving, reduced time of analysis, and mostly a small fluid expenditure for biological, chemistry, and medical applications [1, 3, 7]. Within this context, lab-on-chip devices are being also used with the aim to detect and control slug flows into micro-channel, both in biomedical fields and chemical processes [5-8]. The term two-slug flow refers to two immiscible fluids, which can be gas-liquid, immiscible liquid-liquid or liquid and microparticles, one dispersed in the other, and circulating into the same microsystem $[9,10]$. Among the methods present in literature to control slug flows, the optical one turns out to be advantageous because allows to run an ample range of measurements being minimally invasive [9].

The first microfluidic devices were produced by means of deposition, microfabrication photolithography, and etching processes. Silicon, quartz, and glass were used at first for manufacturing [11-15]. However, many disadvantages were experienced with these processing and materials approaches. These included high raw materials costs, time-consuming and expensive processes needing cleanrooms and, eventually, limited channels geometries. To overcome all these limits, polymers were introduced since they are cheaper than glass or silicon [16], suitable for mass production and biocompatible. Furthermore, polymers display good gas permeability, which is fundamental for biochemistry applications, together with a satisfying optical transparency, which is necessary to implement optical detection methods [17-22].

Among all the polymer types tested in the past decade poly-dimethylsiloxane 
(PDMS) has emerged as the preferred choice [23]. PDMS can be easily moulded by casting to create micro and nano-structures. This fabrication process is also called soft lithography. PDMS has relevant good properties, like nontoxicity, low temperature curing, transparency down to $280 \mathrm{~nm}$ which enable the use for optical detection, good gas permeability and chemically inertness. However, the use of this material has some disadvantages as well. First, once the device is fabricated, it is possible to face variations in size due to the exposure to nonpolar organic solvents. These variations are caused by the swelling phenomena. Second, being the PDMS quite chemical inert, most molecules or polymers will not stick permanently to its surface [1]. This limits the possibility to assemble complex structures because of poor adhesive bonding. Third, PDMS softness and thermal expansion make difficult to obtain high dimensional accuracy [24]. From the processing point of view, even if the soft lithography is a low-cost process, it takes a long time to produce a device, since it requires several processing steps for the manufacturing: fabrication of the master mould, PDMS liquid polymer mixing preparation, casting procedure, PDMS curing, and demoulding [1]. To overcome all these weaknesses the use of a novel 3D printing approach to manufacture in one step only the final device is proposed. The 3D printing process technology used is a vat polymerization approach named Projection Micro Stereolithography $(\mathrm{P} \mu \mathrm{SL})$ patented by the company BMF (www.bmf3d.com). P $\mu$ SL shows several advantages: short turn-around time; down to micron precision; complex shape printing. The design process is simple because the device CAD design can be modeled by software and then printed with no need for mold making and resin's casting [1]. This is a key advantage over soft lithography as it allows to standardize the processing approach removing manual steps which are present in the resin's casting route. Furthermore, when the design concept integrates both the micro-opting and the micro-fluidic items, 3D printing can be used to produce the device in a single fabrication process $[25,26]$.

In this study, a low-cost portable device designed by the authors [27, 28], was manufactured using PDMS for soft lithography and a photocurable resin for the $\mathrm{P} \mu \mathrm{STL}$ manufacturing technique. The $3 \mathrm{D}$ printed device was manufactured in one piece with no assembly using a one-step manufacturing. The designed devices integrated both micro-optic and micro-fluidic components for slug flow detection. In the paper the comparison between the soft lithography and the $\mathrm{P} \mu \mathrm{STL}$ manufacturing processes was reported first. The two materials used for the device manufacturing were then analyzed in terms of their surface's roughness and water angle contact. The setting parameters for optical slug flow detection with the 3D printed device were optimized by using a replicated general factorial design with the aim to identify the optimal test procedure parameters. Once the optimal testing conditions were selected a detailed comparison between the two devices were run to identify the main pros and cons of each system. 


\section{Materials and device processing techniques}

\subsection{Materials}

The master slave for PDMS casting was printed using two materials: VeroWhitePlus RGD835 and FullCure705. RGD835 was purchased from OVERMACH S.p.A. (Parma, Italy) and it is a proprietary blend of acrylate monomers and photoactivators. FullCure705 was purchased from OVERMACH S.p.A. (Parma, Italy) and, as for RGD835, it is a proprietary blend of acrylate monomers and photoactivators. FullCure705 is a breakaway support which is removed by water jetting once the printing process is accomplished. The breakaway support is not part of the final device and is only needed to print not supported cavities.

The master slave was 3D-Printed on a 3D Printer Stratasys Objet260 Connex1 (Stratasys, Los Angeles, CA, USA) and it was used as mold to cast the Device 1. For casting Device 1 the SYLGARDTM 184 Silicone Elastomer kit, composed by a Polymethyl Siloxane (PDMS) elastomer and a suitable curing agent, was purchased from Farnell Italia S.R.L. (Lainate, MI). The procedure used for the manufacturing is detailed elsewhere [28].

HTL Resin is a high performance, rigid and heat resistant yellow resin proprietary developed by Boston Micro Fabrication (Maynard, Massachusetts). HTL was used to manufacture the Device 2 printing on the microArch ${ }^{\circledR} \mathrm{S} 140$ ultra-high resolution $(10 \mu \mathrm{m}) 3 \mathrm{D}$ Printer (Boston Micro Fabrication, Maynard, Massachusetts). This resin grade was selected because of its temperature stability and mechanical properties making it suitable for autoclave sterilization. The resin has, according to its technical data sheet (TDS), a heat distortion temperature (HDT) equal to $140.7^{\circ} \mathrm{C}$ (@0.45MPa) and a Glass Transition Temperature $(\mathrm{Tg})$ of $172^{\circ} \mathrm{C}$. This last parameter is the temperature at which the amorphous regions experience transition from rigid state to more flexible state making the temperature at the border of the solid state to rubbery state [29]. Therefore, the resin is suitable for repeated autoclave cycles making the device suitable for multiple reuses.

\subsection{Micro-Optofluidic device design and working principle}

The micro-optofluidic system that we tested in this work exploits the absorption phenomenon, thus being two different fluids characterized by two different refractive indexes, once an incident laser beam interacts with them, it will be possible to have a different nature of transmission of the light strictly dependent on the fluid with which it is interacting at a precise moment. The working principle is shown in Fig. 1. To practically exploit this physical principle, the geometry of the micro-optofluidic device has been appropriately determined, also thanks to the help of appropriate simulations reported in previous studies [27]. Briefly, the device has two inlets, necessary for the inlet of the two fluids characterizing the two-phase fluid to be studied, which are connected 
to the two micro-channels that, forming a T-junction, guarantee the formation of the two-phase slug flow. At a sufficient distance from the T-junction for the two-phase flow stabilization, with orthogonal orientation to the main microfluidic channel, the micro-channels for the optical fibre's insertion have been designed. From one side of the device the input optical fibre conveyed the laser light, which by crossing through the centre of the microfluidic channel, is then detected by a second optical fibre (the output one), which is aligned with the input one. Using this design, it was possible to acquire a light signal giving direct information about the microfluidic channel inner flow. The flow exits from the main microfluidic channel through an outlet which conveys the fluid to a pressure sensor which can be used as a further detection tool.

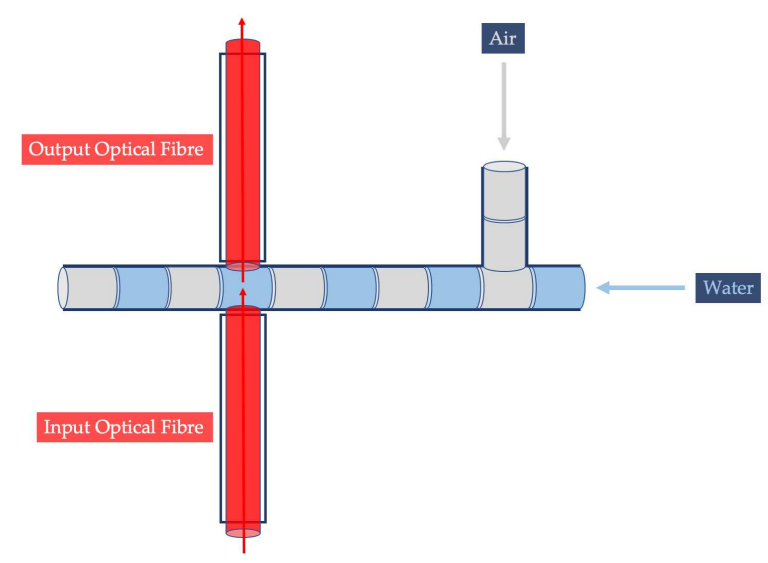

Fig. 1 Working principle of the investigated micro-optofluidic device.

The two devices manufactured by the two processing approaches have the same design in terms of micro-channel dimensions, optical section location, inlet and outlet channels dimension, and T-junction geometry. As shown in Fig. 2, all these features characterize the two devices, although some changes have been made to the 3D printed Device 2, in terms of inlets and outlet positioning, compared to Device 1 made in PDMS [28]. This choice allowed to improve the flow stabilization because of the absence of abrupt direction changes. In fact, even if the inlets and outlet channel's dimension remained unchanged, these are orthogonal to the microfluidic channel in Device 1 and, instead, are coaxial and aligned with the main microfluidic channel in Device 2. The latter design choice for PDMS device is forced by the manufacturing approach that, being based on resin's casting, makes unpractical to realize coaxial inlets and outlets.

\subsection{Micro-Optofluidic devices fabrication processes}

In this study two devices, which differ each other for the fabrication process and the raw materials used, were compared. The first micro-optofluidic system (Device 1) was realized using PDMS with a fabrication process which 


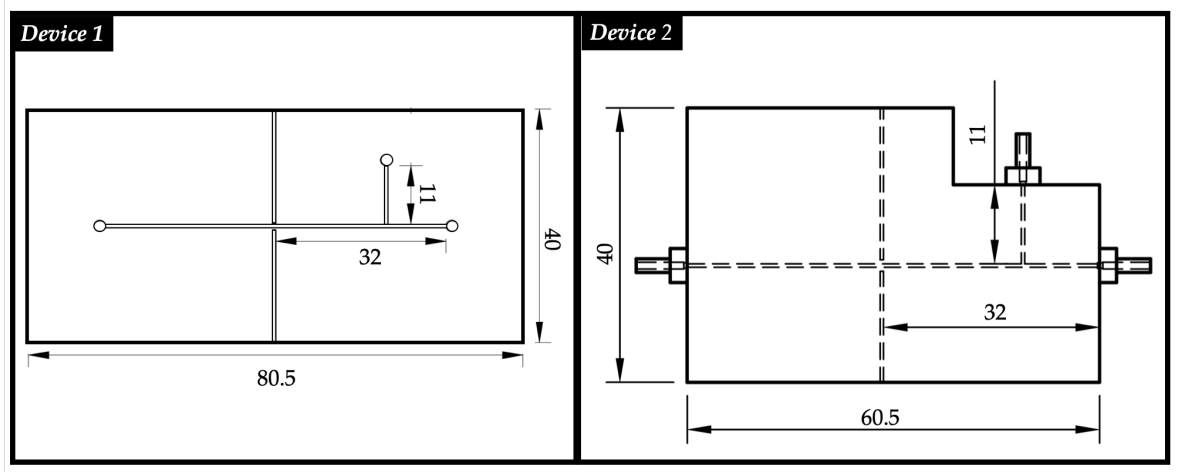

Fig. 2 A comparison from the top view between Device 1 (PDMS) and Device 2 (HTL resin) geometries. Sizes are expressed in $\mathrm{mm}$.

has already been explained elsewhere in detail [27]. The fabrication procedure followed is summarized in Fig. 3.

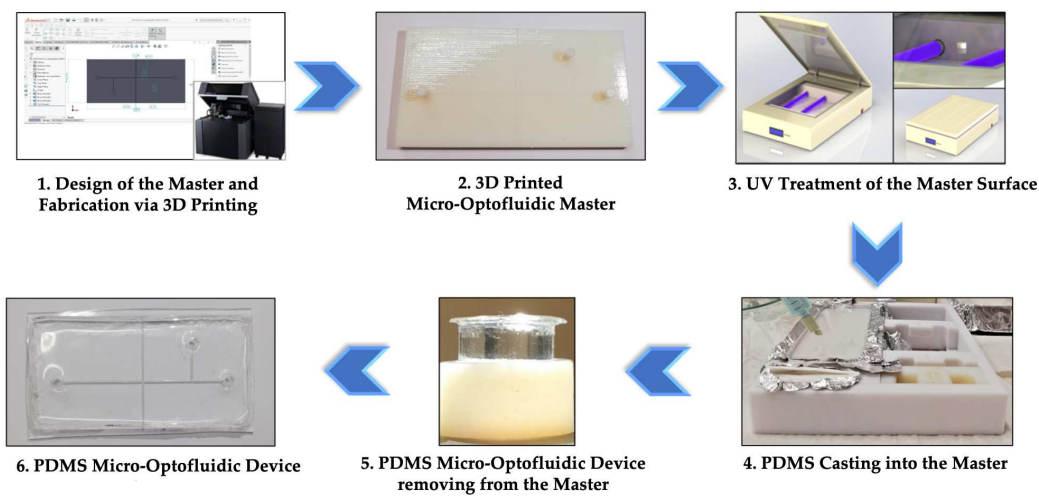

Fig. 3 Procedure followed to fabricate the final Device 1.

The Device 2, conversely, was directly manufactured by using the Projection Micro Stereolithography $(\mathrm{P} \mu \mathrm{SL})$ technique, which allows for ultra-high resolution, accuracy, and precision. $\mathrm{P} \mu \mathrm{SL}$ is a hybrid technique combining the benefits of SLA (Stereolithography) and DLP (Digital Light Processing) 3D Printing techniques. Basically, micro-sized parts are printed in the top down direction, as it happens in the SLA; but the resin is cured as done in DLP, i.e. by using a digital projection screen rather than a small spot laser.

Device 2 was designed using Autodesk ${ }^{\circledR}$ Fusion 360 first and then, the STL file was transferred to the 3D Printer for the build step (Fig. 4). The 
microArch ${ }^{\circledR}$ S140 ultra-high resolution $(10 \mu \mathrm{m}) 3 \mathrm{D}$ Printer was used for the printing.

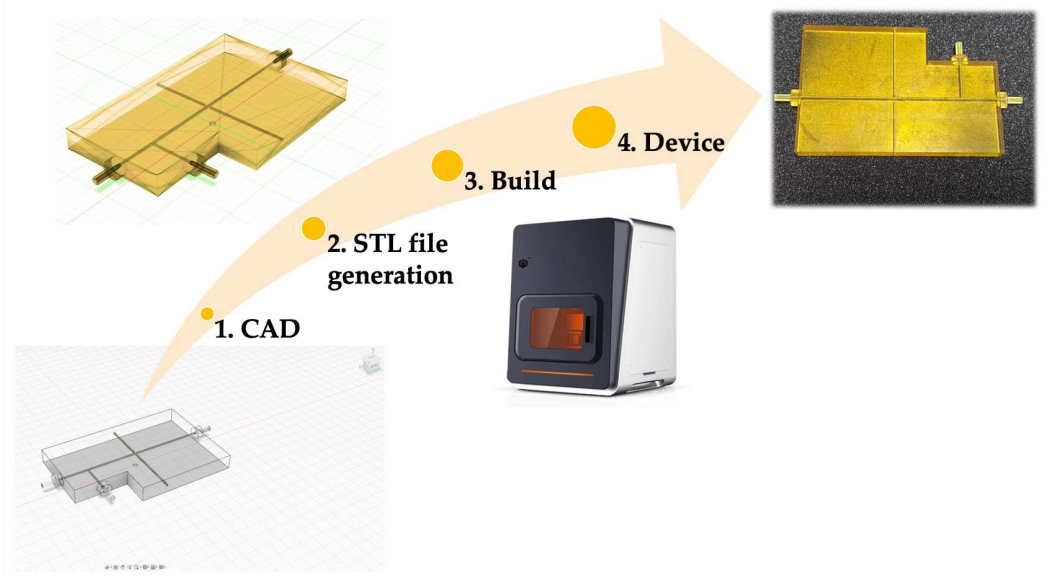

Fig. 4 Steps followed to fabricate the final Device 2 with P $\mu$ SL approach.

The Device 2 being designed and printed as a single piece avoids fluids leakage phenomena or adhesive bonding issues. In addition to that, this processing approach allows to integrate directly the inlets and outlets for fluids flow in the device itself.

\section{Characterization techniques}

\subsection{Slug flow detection setup}

The two-phase slug flow through the micro-optofluidic device has been obtained by simultaneously pumping deionized water and air through the two inlets and the T-junction geometry. In fact, the two inlets have been connected to two different syringe pumps (neMESYS by Cetoni Gmbh), which have been filled with deionized water and air, respectively. The constant flow rate has been varied at three levels: $\{0.1,0.2,0.3\} \mathrm{ml} / \mathrm{min}$.

A laser system (Rgb NovaPro Laser 660-125) with an emission wavelength equal to $600 \mathrm{~nm}$ and an output power ranging between $1 \mathrm{~mW}$ and $3 \mathrm{~mW}$ has been used as the light source aimed at detecting the two phases. Finally, to test the designed micro-optofluidic device three different measurement systems have been considered:

- a photodiode (PDA 100A, Thorlabs, with a gain range between $30 \mathrm{~dB}$ and $40 \mathrm{~dB}$ ) measuring the light intensity variation, connected to a PC oscilloscope (Picoscope 2204A) with a sampling frequency of $1.5 \mathrm{kHz}$; 
- a digital USB microscope placed above the optical acquisition section, that allowed the interactions between the main microfluidic channel and the light transmission to be monitored;

- a pressure sensor (Sensirion, SF1300), having a sampling frequency equal to $200 \mathrm{~Hz}$, monitoring the stability of the upstream fluid flow rate externally to the channel.

A block scheme and a real picture of the experimental setup used for the slug flow detection are shown, respectively, in Fig. 5a and Fig. 5b.

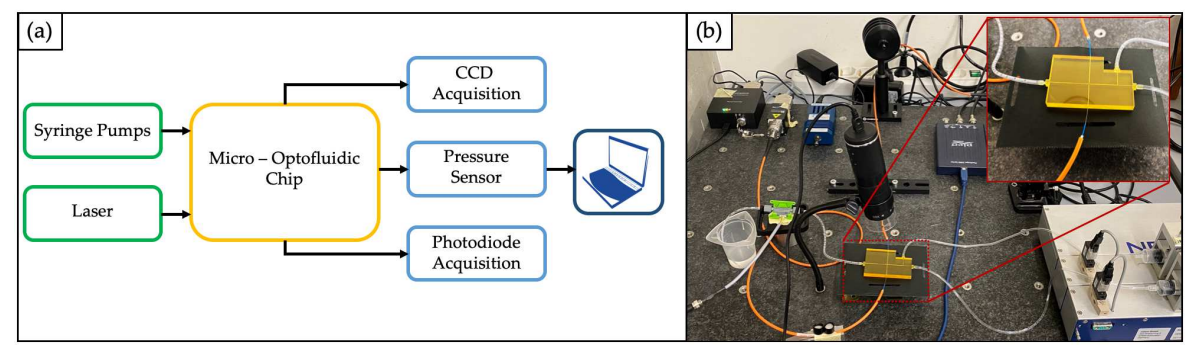

Fig. 5 Experimental setup: (a) block scheme; (b) real picture.

\subsection{The acquired signal and its processing}

The acquired signals by the photodiode is a square wave. This behavior is justified by the different refractive index that characterizes the two fluids generating the tested two-phase flow: the refractive indexes for the air and the water are respectively equal to $n_{\text {Air }}=1.0$ and $n_{\text {Water }}=1.3$.

Under these conditions, the higher level of the square wave signal corresponds to the water slug, while the lower level corresponds to the air slug. Each transition from the high to the low level of the square wave is characterized by the presence of signal peaks depending on the transitory phase between the two fluids fronts. The signal detected by the photodiode is shown in Fig. 6 .

The optical signals need signal smoothing. The signal post-processing was carried out using a Matlab (MathWorks ${ }^{\circledR}$ ) routine as described elsewhere in detail [28]. After the acquisition phase, a low-pass filter with a $40 \mathrm{~Hz}$ cutoff frequency has been applied to saturate the signals, thus eliminating high frequency components. Then, a smoothing procedure has been run to generate perfect square wave signals. By overlying the square wave to the saturated signals, it has been possible to identify the time frames corresponding to the air detection $\left(T_{a}\right)$, and the time frames corresponding to the water detection $\left(T_{w}\right)$ by the device.

In addition, to evaluate the mean period associated to a complete air-water slug passage the optical signal has been analyzed in the frequency domain. In fact, by obtaining the frequency spectrum, at first it was possible to determine 


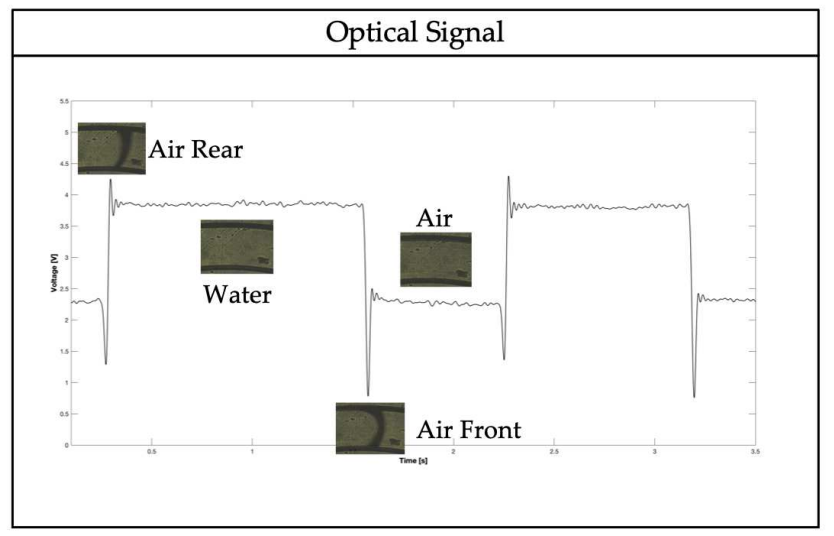

Fig. 6 Optical acquired signal interpretation by acquired CCD video frames.

the frequency peak $\left(f_{p h}\right)$, and then it was possible to determine the mean period $T_{p h}$ by calculating the reciprocal of this parameter.

\subsection{Surface characterization techniques}

Two surface characterization techniques were used: Atomic Force Microscopy (AFM) to characterize surface's roughness and static water angle to measure hydrophilicity. Atomic Force Microscopy (AFM) was carried out by mean of an AFM NTEGRA by NT-MDT. The test was run in semi-contact mode, with a rate equal to $0.5 \mathrm{~Hz}$, and by using a tip HA-NC (ETALON) characterized by a resonant frequency of $140 \pm 10 \% \mathrm{kHz}$. Eventually, in the AFM analysis a $5 \times 5$ $\mu m^{2}$ area was investigated. For each analysis three parameters were evaluated, which are the roughness distribution in the square surface (RMS), average roughness (RA), and the peak to peak parameter. All the surfaces characterized were obtained from the processing methods reported above without any further treatment.

Static water contact angles $(\theta)$ measurements were run to evaluate the surface hydrophilicity of the two materials used. A Lite Optical Tensiometer TL100 with an accuracy of $\pm 3^{\circ}$, at room temperature and in air atmosphere was used for the experiments. The test was carried out as it follows: first, $5 \mu \mathrm{L}$ of Milli$\mathrm{Q}$ water drop, having a resistivity equal to $18.2 \mathrm{M} \Omega$ at $25^{\circ} \mathrm{C}$, was deposited on the device's surface with a calibrated micro-syringe; next, measurements of $\theta$ were made on both sides of the two-dimensional projection of the droplet. Five different sets of measurements have been performed on different surface portions of every sample to obtain statistically reliable results. 


\section{Results and Discussion}

\subsection{Surface analysis}

For many applications in microfluidic technology the behavior at the solidliquid interface is of prime importance. For example, changes in the fluid wetting ability, may affect the exchange momentum of the fluid with the solid surface at the atomic scale, resulting in a velocity slip at the solid wall. Thus, considering that in this paper water is the main flowing fluid, it is important to measure the solid surfaces behavior. To be more specific, hydrophilic surfaces are more attractive toward water since, being they bear electric charges or polar groups, water molecules interact favorably with them. Instead, hydrophobic surfaces are water unattractive because, being them non polar, they cause water molecules to lack of hydrogen bonding partners [30, 31]. Moreover, the flow characteristics in micro-channels may be different from the classical theory for macro-channels. Many experimental and numerical results demonstrated that the friction factor is much higher in micro-channels than in macro-ones [32]. In this context, the surface roughness is of fundamental importance for the understanding of these deviations, since by reducing the channel dimensions, the roughness importance increases. For all these reasons, both the static water contact angle and surfaces roughness must be evaluated to gain a complete understanding of the hydrodynamic nature of the two-phase slug fluid into the microchannels of the two studied devices.

The surface roughness of the PDMS and HTL resin was evaluated using Atomic Force Microscopy (AFM). In AFM, the probe tip is fixed to a cantilever beam. The probe interacts with the surface and the resulting force deflects the beam in a repulsive manner, as described by Hooke's Law. In the same manner that a spring changes dimensions under the influence of forces, the attractive and repulsive forces between atoms of the probe and the surface can also be monitored when brought extremely close to each other. Hence, the net forces acting on the probe tip deflect the cantilever, and the tip displacement is proportional to the force between the surface and the tip. As the probe tip is scanned across the surface, a laser beam reflects off the cantilever. By monitoring the net $(\mathrm{x}, \mathrm{y}$, and $\mathrm{z}$ ) deflection of the cantilever, a three-dimensional image of the surface is constructed [33].

The obtained results from AFM analysis are shown in Fig. 7 for the PDMS and in Fig. 8 for the HTL resin.

The surface roughness of the HTL resin is higher than the PDMS ones (Tab. 1), in fact both the RMS and the RA parameters evaluated for the HTL resin are the $97 \%$ higher than the PDMS ones. Moreover, according to the results, while for the HTL resin a thick web of filaments was spotted on the surface, the PDMS one appeared strongly flatter. For PDMS the analysis revealed the presence of some scattered nanometric bubbles which can origin from entrapped air or residual solvent.

The relative roughness of the microfluidic channels can be measured as the ratio between the RA parameter and the side of the channel (this being a 


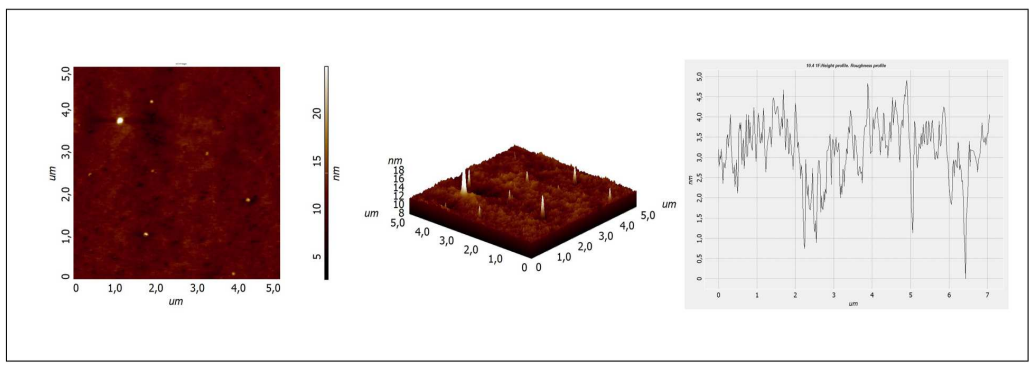

Fig. 7 Roughness measurements of PDMS surface.

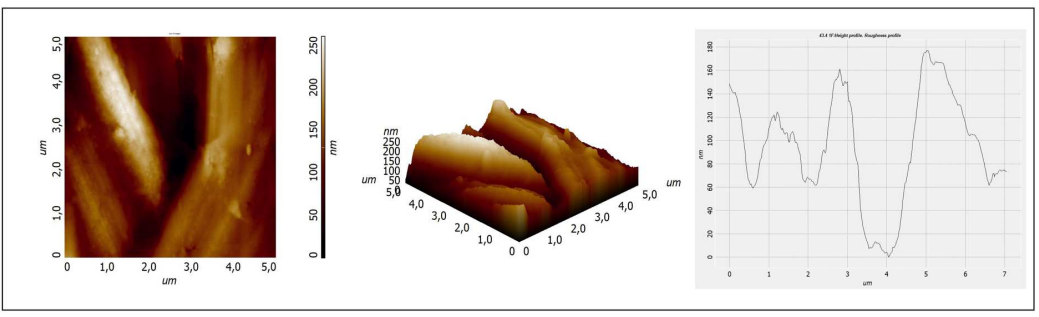

Fig. 8 Roughness measurements of HTL resin surface.

Table 1 AFM analysis results.

\begin{tabular}{cccc}
\hline $\begin{array}{c}\text { Material } \\
\text { Investigated }\end{array}$ & $\begin{array}{c}\text { RMS } \\
{[\mathbf{n m}]}\end{array}$ & $\begin{array}{c}\text { RA } \\
{[\mathbf{n m}]}\end{array}$ & $\begin{array}{c}\text { Peak to Peak } \\
{[\mathbf{n m}]}\end{array}$ \\
\hline PDMS & 1.097 & 0.763 & 35.158 \\
HTL resin & 47.056 & 37.324 & 259.121 \\
\hline
\end{tabular}

square section). This parameter can be used to evaluate the flow stability in the microchannel. For the studied surfaces the relative roughness was equal to $0.0001 \%$ and $0.0002 \%$ for PDMS and HTL resin, respectively. In other papers, relative roughness values of the order of magnitude of the micrometer were reported showing, for the devices, an increase of $8-38 \%$ for the fiction factors over the classical theory $[34,35]$. Therefore, the values measured here let to conclude that there will be no instability of the flow inside the channel, i.e. flow slowdown or inhibition, for the manufactured devices. These measurements showed that both the manufacturing techniques used can guarantee a stable flow inside the microchannel.

The water angle contact measurements are shown in Tab. 2. The contact angle for the PDMS was equal to $111.84^{\circ}$, while the HTL resin presented a value of $64.36^{\circ}$. 
Table 2 Contact angles measurements results.

\begin{tabular}{cc}
$\begin{array}{c}\text { Material } \\
\text { Investigated }\end{array}$ & $\begin{array}{c}\text { CA } \\
{\left[{ }^{\circ}\right]}\end{array}$ \\
\hline PDMS & 111.84 \\
HTL resin & 64.36 \\
\hline
\end{tabular}

The micro-optofluidic devices have been tested by using an aqueous two-phase system. In some previous studies high water angle contacts showed advantageous results because when the aqueous droplets encounter a wall, they bounce off instead of sticking [36]. In other studies, low water contact angles resulted in some flow complications due to the adhesion of water droplets to the wall $[37,38]$. In order to unveil issues with flow advancement and process stability within the channel, slug flow detection set up for Device 2 was studied as reported in the following.

\subsection{Optimization of the slug flow detection set up for the 3D printed device (Device 2)}

To optimize the slug flow detection set up for the 3D printed device (Device 2) a replicated general factorial design was carried out. Two design factors (independent variables) have been considered for the test procedure: the laser input power (factor A) and the fluid flow rate (factor B). While factor A was varied at two levels $(a=2)$, factor $\mathrm{B}$ was varied at three levels $(b=3)$. The $a=2$ levels correspond to $1 \mathrm{~mW}$ and $5 \mathrm{~mW}$, respectively. The $b=3$ levels correspond to $\{0.1,0.2,0.3\} \mathrm{ml} / \mathrm{min}$. The number of replications has been set equal to $n=3$, for a total of $N=a \cdot b \cdot n=18$ experimental runs. The experimental plan is summarized in Tab. 3 .

Table 3 Experimental plan: factors and levels investigated.

\begin{tabular}{lcccccc}
\hline Factor & Symbol & Type & Unit & $\begin{array}{c}\text { Low Level } \\
(\mathbf{- 1})\end{array}$ & $\begin{array}{c}\text { Center Level } \\
\mathbf{( 0 )}\end{array}$ & $\begin{array}{c}\text { High Level } \\
(+\mathbf{1})\end{array}$ \\
\hline $\begin{array}{l}\text { Laser Input } \\
\text { Power }\end{array}$ & A & Numerical & {$[\mathrm{mW}]$} & 1 & - & 5 \\
\hline Flow Rate & B & Numerical & {$[\mathrm{ml} / \mathrm{min}]$} & 0.1 & 0.2 & 0.3 \\
\hline
\end{tabular}


Next, the following responses (dependent variables) have been considered for the experimental plan:

- the voltage difference $(\Delta V)$, defined as the difference between the grand averages of the observations measured at the high and the low level of the square wave optical signal, respectively. The calculation of the voltage difference $(\Delta V)$ is as follows. By starting from the optical signal acquisition for a period of $15 \mathrm{~s}$, the signal is post-processed as previously described. Next, in each acquired and post-processed optical signal the sequence of high-level values in the wave square is collected in a time order, thus getting a single sample of $V_{h}(i)$ observations, for $i=1, \ldots, N_{h}$. The same procedure is performed for the low levels of the square wave, thus obtaining a single sample of $V_{l}(i)$ observations, for $i=1, \ldots, N_{l}$. Here $N_{h} \in\left(2,5 \cdot 10^{3}, 10^{4}\right)$ and $N_{l} \in\left(2,5 \cdot 10^{3}, 10^{4}\right)$. Next, the corresponding sample mean values $\left(\bar{X}_{h}\right.$ and $\left.\bar{X}_{l}\right)$ and sample standard deviations $\left(s_{h}\right.$ and $\left.s_{l}\right)$ are evaluated for each sample $V_{h}(i)$ and $V_{l}(i)$, respectively. Having both $V_{h}(i)$ and $V_{l}(i)$ a very large number of observations, both their sample means $\left(\bar{V}_{h}\right.$ and $\left.\bar{V}_{l}\right)$ are approximately normally distributed random variables with estimated parameters:

$$
\begin{gathered}
\bar{V}_{h} \sim N\left(\bar{X}_{h}, \frac{s h_{h}{ }^{2}}{N_{h}}\right) \\
\bar{V}_{l} \sim N\left(\bar{X}_{l}, \frac{s_{l}{ }^{2}}{N_{l}}\right)
\end{gathered}
$$

Starting from this assumption, the response $\Delta V$ is a normal random variable having distribution:

$$
\Delta V=\bar{V}_{h}-\bar{V}_{l} \sim N\left(\bar{X}_{h}-\bar{X}_{l}, \frac{s_{h}^{2}}{N_{h}}+\frac{s_{l}^{2}}{N_{l}}\right)
$$

- The second investigated response is the mean period $\left(T_{p h}\right)$ associated to a complete air-water slug passage, obtained as the reciprocal of the frequency peak gained from the amplitude spectrum of the acquired optical signal:

$$
T_{p h}=\frac{1}{f_{p h}}=<T_{w}>+<T_{a}>\text {. }
$$

In particular, the first response $\Delta V$ is considered to show that the optical part of the device is really able to discriminate between the two fluids that make up the two-phase slug flow. While, the second response $T_{p h}$ is taken into account to evaluate if a real hydrodynamic stability is achieved into the micro-channel. Once the response observations have been calculated, an Analysis of Variance (ANOVA) study has been performed to investigate the statistical significance of each factor and their interaction. The observations regarding the first response, for all the investigated scenarios and all the run replications, are summarized in Tab. 4. While the ANOVA table for the $\Delta V$ response and a bar plot comparing the various cases are shown in Tab. 5 and Fig. 9, respectively. 
Table $4 \Delta V$ observations [V] for the investigated scenarios. Factors: Input Power $(\mathrm{P})$ and Flow Rate (FR). $\mathrm{P}=\{1,5\} \mathrm{mW} ; \mathrm{FR}=\{0.1,0.2,0.3\} \mathrm{ml} / \mathrm{min}$.

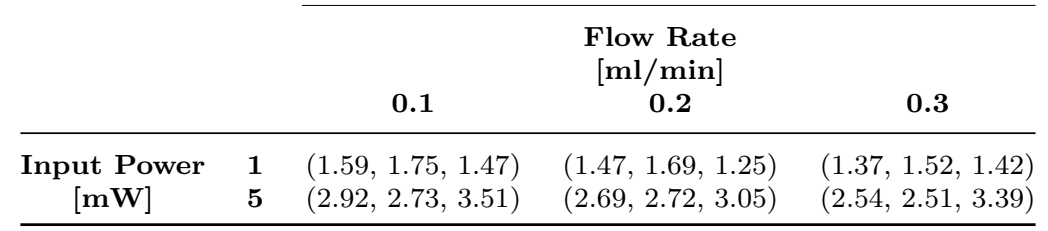

Table 5 ANOVA table for the response voltage difference $(\Delta V)$.

\begin{tabular}{lccccc}
\hline \multicolumn{1}{c}{ Source } & Sum of Squares & df & Mean Square & F Value & $\boldsymbol{p}$-Value \\
\hline Model & 8.854417 & 2 & 4.427208 & 60.70683 & $<0.0001$ \\
A-Input Power & 8.72826 & 1 & 8.72826 & 119.6838 & $<0.0001$ \\
B-Flow Rate & 0.126157 & 1 & 0.126157 & 1.729892 & 0.2082 \\
Residual & 1.093915 & 15 & 0.072928 & & \\
$\quad$ Lack of Fit & 0.035664 & 3 & 0.011888 & 0.134802 & 0.9374 \\
$\quad$ Pure Error & 1.058252 & 12 & 0.088188 & & \\
Cor Total & 9.948332 & 17 & & & \\
\hline R-Squared & 0.8900 & & & & \\
Adj R-Squared & 0.8754 & & & & \\
\hline
\end{tabular}

From the ANOVA study shown in Tab. 5, the laser input power (factor A) is the only influential factor $(p$-value $<0.001)$ on voltage difference. Both the $\mathrm{R}$ squared and the adjusted R-squared have high values $\left(R^{2}=0.89, R_{a d j}^{2}=0.87\right)$, thus most of the variability in the observations is explained by the laser input power. From the model adequacy checking on the residuals, no anomalies were identified. Deeper investigation on the obtained results shows that by increasing the input power of the laser, the gap existing between the averages at the high and low levels of the square wave optical signal almost doubles. This results in a higher discrimination power of Device 2 between air and water. However, increasing the input power leads to a slightly higher dispersion in the observations. When the laser power is at the low level, the discrimination power is still sufficient. Therefore, it is convenient to design a higher laser input power for all those scenarios where the difference between the refractive indexes of the two investigated fluids is narrow. 


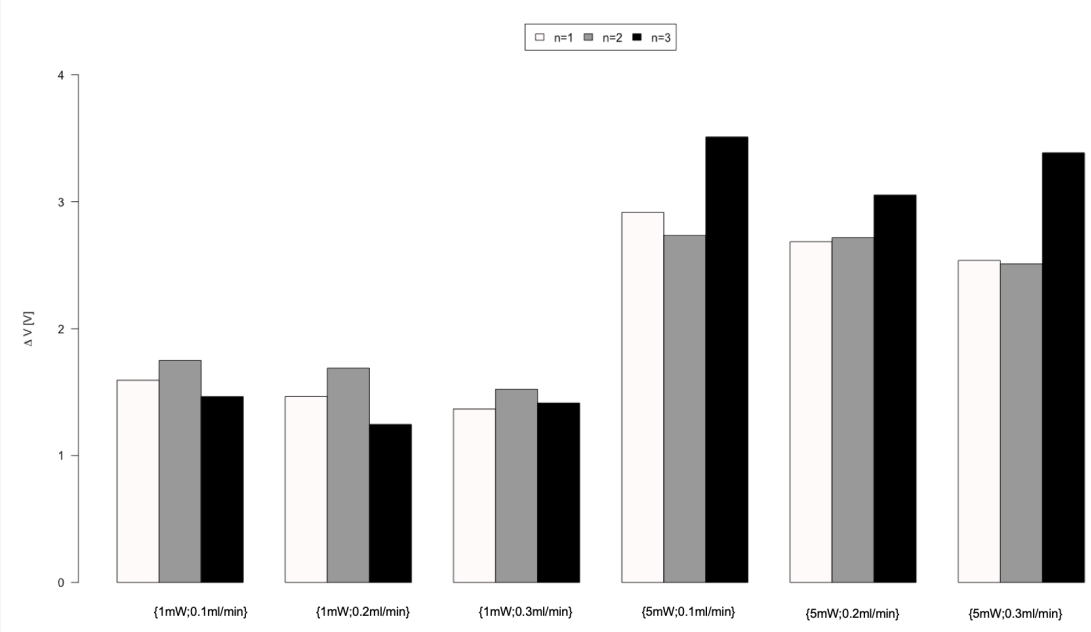

Fig. 9 Bar plot showing the voltage difference $(\Delta V)$, for each investigated scenario and replication.

The observations obtained for the mean period $\left(T_{p h}\right)$ are summarized in Tab. 6 and shown in Fig. 10. The results of the ANOVA study are shown in Tab. 7.

Table $6 T_{p h}$ observations for the investigated scenarios. Factors: Input Power $(\mathrm{P})$ and Flow Rate (FR). $\mathrm{P}=\{1,5\} \mathrm{ml} / \mathrm{min} \mathrm{mW} ; \mathrm{FR}=\{0.1,0.2,0.3\} \mathrm{ml} / \mathrm{min}$.

\begin{tabular}{ccc}
\hline & $\begin{array}{c}\text { Flow Rate } \\
{[\mathbf{m l} / \mathbf{m i n}]} \\
\mathbf{0 . 2}\end{array}$ & $\mathbf{0 . 3}$ \\
\hline $\mathbf{0 . 1}$ & & \\
$(1.88,1.87,1.14)$ & $(0.60,0.94,0.60)$ & $(0.37,0.50,0.47)$ \\
$(1.87,1.87,1.13)$ & $(0.63,0.91,0.60)$ & $(0.37,0.65,0.47)$ \\
\hline
\end{tabular}

The flow rate (factor B) is the only statistically influencing factor on the mean period, as shown in the ANOVA study (Tab. 7). Although the parameter laser input power (factor A) is clearly not influential ( $p$-value $>0.001$ ) on the mean period, it has been considered in the study to account for the possibility that at higher power values, the fluid would be heated more, with a consequent lower viscosity value and a higher fluid velocity. No anomalies have been found from the model adequacy checking on the residuals. Both the R-squared and the adjusted $\mathrm{R}$-squared have quite high values $\left(R^{2}=0.75, R_{a d j}^{2}=0.71\right)$. The flow stability is significantly improved at higher values of the flow rate, thus suggesting designing a flow rate equal to $0.3 \mathrm{ml} / \mathrm{min}$.

Summarizing the results obtained from the two responses, it is reasonably legitimate to assume that a good test configuration for the micro-optofluidic device should consider $(\mathrm{P}, \mathrm{FR})=(5 \mathrm{~mW}, 0.3 \mathrm{ml} / \mathrm{min})$.

The optical signals and the corresponding amplitude spectrums related to 
Table 7 ANOVA table for the response mean period $\left(T_{p h}\right)$.

\begin{tabular}{cccccc}
\hline Source & Sum of Squares & df & Mean Square & F Value & p-Value \\
\hline Model & 4.001519 & 2 & 2.00076 & 21.92583 & $<0.0001$ \\
A-Input Power & 0.000931 & 1 & 0.000931 & 0.010199 & 0.9209 \\
B-Flow Rate & 4.000589 & 1 & 4.000589 & 43.84147 & $<0.0001$ \\
Residual & 1.368769 & 15 & 0.091251 & & \\
Lack of Fit & 0.458317 & 3 & 0.152772 & 2.013578 & 0.1658 \\
Pure Error & 0.910452 & 12 & 0.075871 & & \\
Cor Total & 5.370288 & 17 & & & \\
\hline R-Squared & 0.7451 & & & & \\
Adj R-Squared & 0.7111 & & & & \\
\hline
\end{tabular}

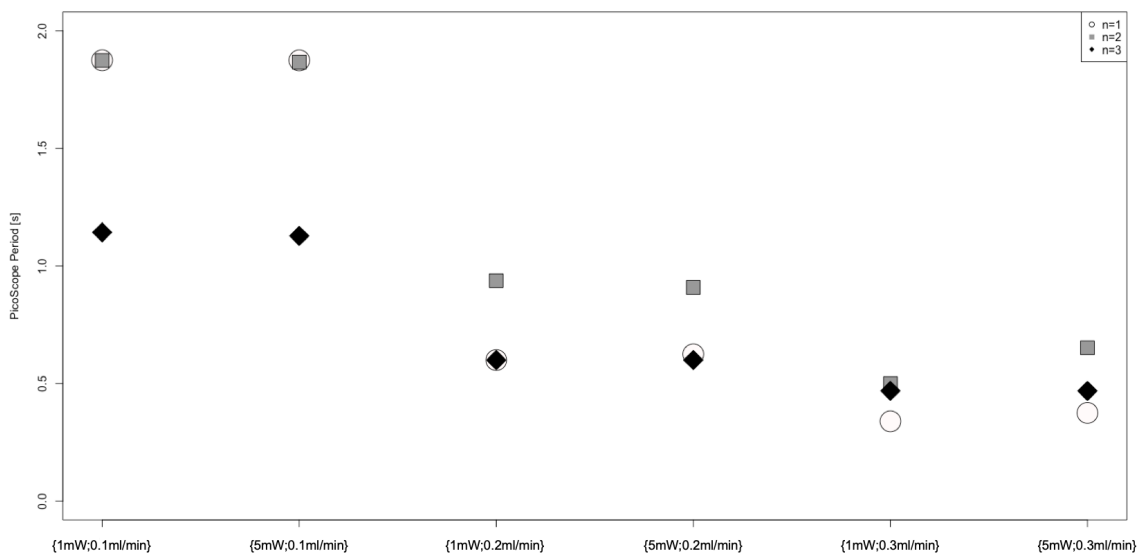

Fig. 10 Trend related to the mean period $\left(T_{p h}\right)$ associated to a complete air-water slug passage for each investigated scenario and replication. $n=1,2,3$ refer to the three run replications.

the optimal operating condition are shown in Fig. 11. Looking at them, it is possible to discriminate each fluid of the two-phase slug. Even though the discrimination between air and water is always possible, not always the maximum and minimum level of the square wave optical signals are at the same level by repeating the signal acquisition under the same conditions. This phenomenon can be attributed to centering problems between the two optical fibers (input and output) since, once inserted in the appropriate device's slots, a little mechanical backlash occurs. Nevertheless, what matters most is that, by focusing on the first investigated response (i.e. voltage difference $\Delta V$ ), it was spotted a reasonable repeatability at the same process condition for all the replications run. 


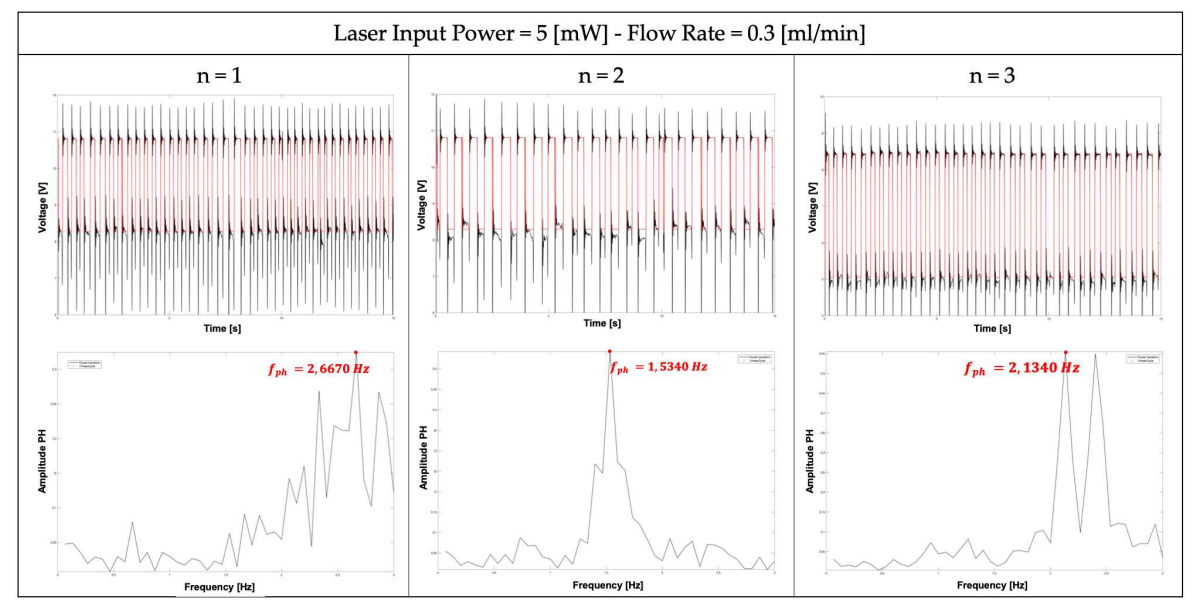

Fig. 11 Upper Row: optical signals in the time domain for each replication $(n=1,2,3)$ with $(\mathrm{P}, \mathrm{FR})=(5 \mathrm{~mW}, 0.3 \mathrm{ml} / \mathrm{min})$. Lower Row: optical signals in the frequency domain for each replication $(n=1,2,3)$ with $(\mathrm{P}, \mathrm{FR})=(5 \mathrm{~mW}, 0.3 \mathrm{ml} / \mathrm{min})$. The three identified peaks are necessary to evaluate the mean period $\left(T_{p h}\right)$ associated to a complete air-water slug passage.

\subsection{Comparative analysis between Device 1 and Device 2}

Once the optimal test configuration was identified for the Device 2, a comparison was carried out between the two devices. The aim is to identify the most performing device and the one affected by the lower repeatability error. The comparison between the 3D printed device and the PDMS device [28] was carried out for all the investigated responses. The chosen test set up for the comparison was $(\mathrm{P}, \mathrm{FR})=(5 \mathrm{~mW}, 0.3 \mathrm{ml} / \mathrm{min})$. This decision is justified by the fact that setting the flow rate equal to $0.3 \mathrm{ml} / \mathrm{min}$ showed to be the best operating condition for improving the flow stability while, choosing the laser power equal to $5 \mathrm{~mW}$ allowed to have the highest discrimination between air and water levels.

The results relating to the voltage difference $(\Delta V)$ are summarized in Tab. 8 and in Fig. 12. The $\Delta V$ parameter is always higher for the Device 1 compared to the Device 2. This result means that Device 1 better discriminates the two investigated fluids. This result is justifiable by the fact that while the PDMS is fully transparent, the HTL resin is not. However, the dispersion of observations collected by using the Device 2 is much smaller than Device 1. This result can be explained by considering that, while the Device 2 is manufactured with a high stiffness resin, the Device 1 is made of a flexible material, which can lead to difficulties in centering the two optical fibers resulting in higher variations for the measurements obtained.

Once again, consistently with what has been said so far, the same reasoning can be made for the second investigated response, i.e. the mean period $T_{p h}$. In fact, accordingly with the results summarized in Tab. 9 and shown in Fig. 13, 
Table 8 A comparison between the Device 1 and the Device 2 regarding the Voltage difference $(\Delta V)$.

\begin{tabular}{cccccc}
\hline & & & $\mathbf{n}=\mathbf{1}$ & $\mathbf{n}=\mathbf{2}$ & $\mathbf{n}=\mathbf{3}$ \\
\hline ID & Flow Rate & Input Power & $\Delta V$ & $\Delta V$ & $\Delta V$ \\
{$[-]$} & {$[\mathbf{m l} / \mathbf{m i n}]$} & {$[\mathbf{m W}]$} & {$[\mathbf{V}]$} & {$[\mathbf{V}]$} & {$[\mathbf{V}]$} \\
Device 1 & 0.3 & 5 & 7.80 & 6.29 & 9.73 \\
Device 2 & 0.3 & 5 & 2.54 & 2.51 & 3.39 \\
\hline
\end{tabular}

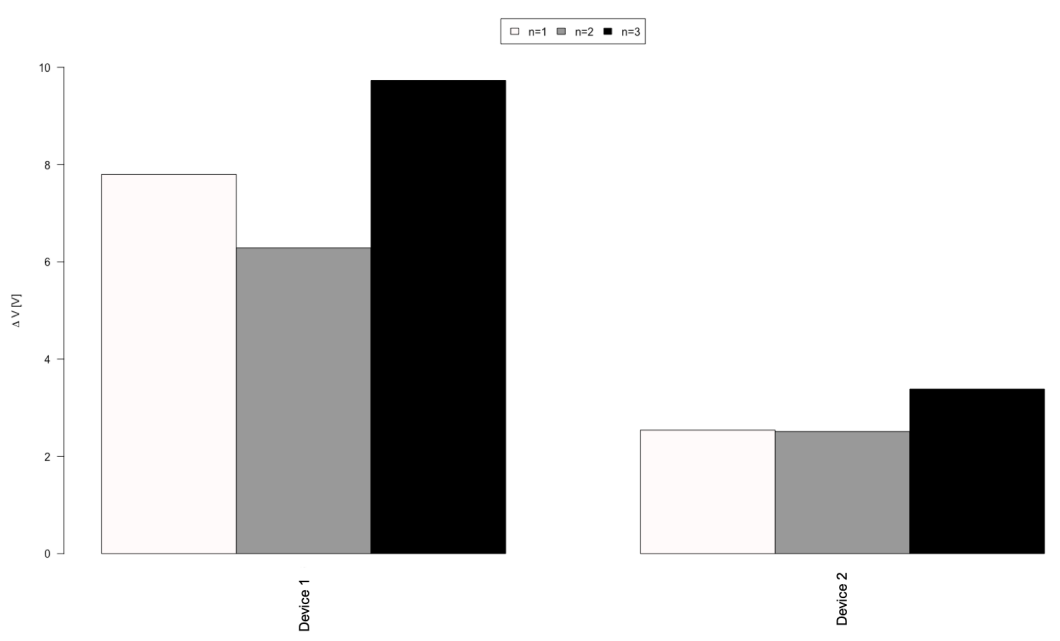

Fig. 12 Bar plot comparing the Device 1 and the Device 2 with respect to the first investigated response, i.e. the Voltage Difference $(\Delta V)$, for each replication.

the observed data for the Device 1 present a higher dispersion than Device 2. Thus, Device 2 shows a greater flow stability than Device 1. Moreover, it is possible to assume that the HTL resin surface properties allow for a faster two-phase slug flow than the PDMS device. Indeed, the mean period value for the Device 2 is approximately equal to $12 \%$ the value for Device 1 .

Table 9 A comparison between the Device 1 and the Device 2 regarding the Mean Period associated to a complete air-water slug passage $\left(T_{p h}\right)$.

\begin{tabular}{ccccccc}
\hline & & & $\mathbf{n}=\mathbf{1}$ & $\mathbf{n}=\mathbf{2}$ & $\mathbf{n}=\mathbf{3}$ & \\
\hline ID & Flow Rate & Input Power & $\mathbf{T}_{\mathbf{p h}}$ & $\mathbf{T}_{\mathbf{p h}}$ & $\mathbf{T}_{\mathbf{p h}}$ & Mean \\
{$[-]$} & {$[\mathbf{m l} / \mathbf{m i n}]$} & {$[\mathbf{m W}]$} & {$[\mathbf{s}]$} & {$[\mathbf{s}]$} & {$[\mathbf{s}]$} & {$[\mathbf{s}]$} \\
Device 1 & 0.3 & 5 & 4.95 & 4.29 & 4.09 & 4.09 \\
Device 2 & 0.3 & 5 & 0.37 & 0.65 & 0.50 & 0.50 \\
\hline
\end{tabular}




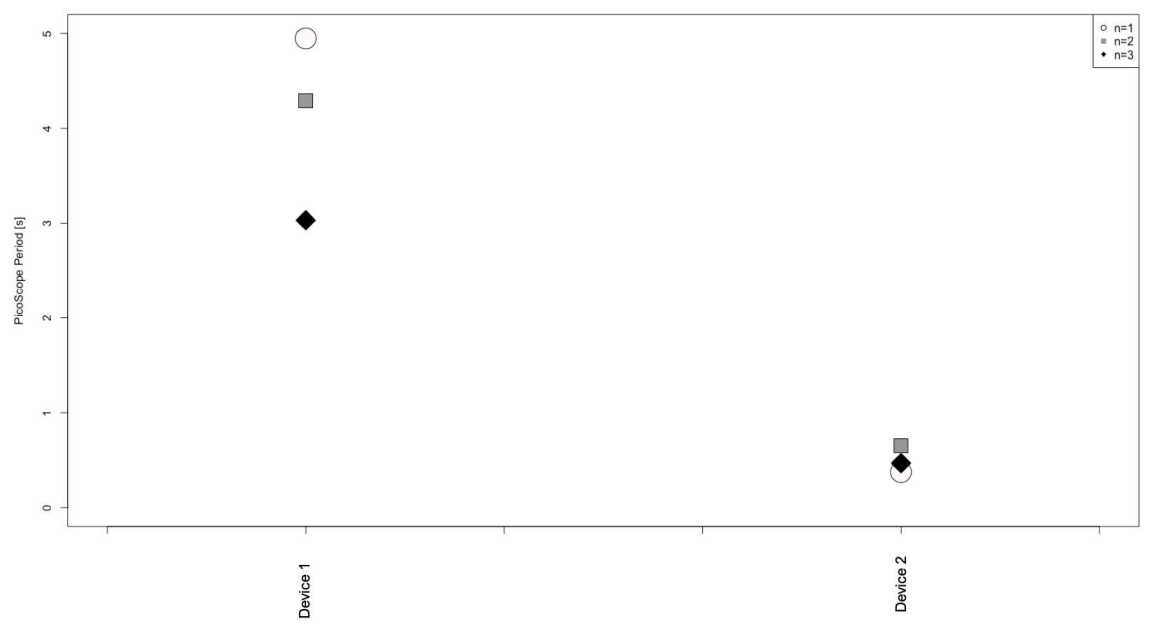

Fig. 13 Comparison of the trend related to the mean period $\left(T_{p h}\right)$ associated to a complete air-water slug passage between the Device 1 and the Device 2.

Although the results relating to this last comparison may appear contradictory with what has been said previously regarding the chemical nature of the surfaces identified, since the flow is slower in the PDMS device than in the HTL resin one, a further important aspect must be considered. The design of the macro-to-micro interface, that is the inlets connecting the micro-optofluidic device to the fluids loading tubes, implemented in the two analyzed devices is different. In fact, while the vertical (top-loading) inlet approach was used for the Device 1, the parallel (in-line) ones was used for the Device 2. According with previous study run for similar flow velocity range [39], the design decision for the 3D printed device made it possible to obtain a maximum speed in the center of the channel and along itself. Instead, the approach used for the inlets in Device 1 was able to obtain a maximum flow speed only in correspondence of the macro-to-micro interface, which adequately justifies the obtained results.

\section{Conclusion}

In this work two micro-optofluidic flow devices were manufactured and fully characterized. A novel approach based on the Projection Micro Stereolithography $(\mathrm{P} \mu \mathrm{SL}) 3 \mathrm{D}$ printing approach was compared to the casting procedure developed by the authors [28]. The 3D printing approach selected allowed to obtain in one-step the final device skipping all in once all the manual steps which are required by the casting procedure. In addition to that, the possibility to design and print complex geometries allowed to fully integrated coaxial inlets and outlets thus, overcoming a design limitation of the casting approach. The materials used for the two approaches differed in terms of roughness and 
water angle with the 3D printing material (HTL resin). In particular, the relative roughness for the two materials ranged between $0.0001 \%$ and $0.0002 \%$ for PDMS and HTL resin, respectively. These values mean that no flow instability inside the microchannel would be associated to the solid surface making both the materials suitable, in terms of their surface finishing, for microfluidic devices.

The $3 \mathrm{D}$ printed device was tested for two phase slug flow detection using the same set up used previously for PDMS based devices [28]. The parameters used for the flow detection were optimized using ANOVA to rationalize the effect of each varied parameter on the selected responses. The input laser power and the flow rate levels of $5 \mathrm{~mW}$ and $0.3 \mathrm{ml} / \mathrm{min}$ were found as the best parameters to improve both the discrimination between fluids and the flow stability. The performance of the two devices as two-phase slug flow detector was analyzed using the optimized setup parameters identified. The comparison revealed that the Device 2, being printed with a stiff resin embedding the coaxial inlets and outlets, returned lower dispersed data compared to Device 1. While, Device 1 presented higher $\Delta V$ values resulting in a better discrimination of the two investigated fluids. The findings of the comparative analysis allowed to highlight the advantages and disadvantages of the $3 \mathrm{D}$ printing approach used. The advantages of $\mathrm{P} \mu \mathrm{SL}$ printing are its suitability to print with high accuracy and in one step complex micro-optofluidic devices. However, the use of resin with lower transparency compared to PDMS results in a lower detection performance requiring the use of higher input laser power. Therefore, the future research activity must be driven toward the development of resin's grades for 3D printing showing the same optical properties of PDMS. This goal would enhance the possibility to use $3 \mathrm{D}$ printing to replace PDMS casting.

Acknowledgments. The authors acknowledge the funding received on this project from Universitá degli Studi di Catania under the Grant Scheme PIACERI with the project MAF-moF "Materiali multifunzionali per dispositive micro-optofluidici" Project Coordinator Maria Elena Fragalá.

Gianluca Cicala acknowledges also Italian MIUR grant number 20179SWLKA Project Title Multiple Advanced Materials Manufactured by Additive technologies (MAMMA), under the PRIN funding Scheme.

\section{Declarations}

- Conflict of interest: The authors declare no conflict of interest. The funders had no role in the design of the study; in the collection, analyses, or interpretation of data; in the writing of the manuscript, or in the decision to publish the results. 


\section{References}

[1] Scott SM, Ali Z (2021) Fabrication methods for microfluidic devices: An overview. Micromachines 12. https://doi.org/10.3390/mi12030319.

[2] Zhou W, Le J, Chen Y, Cai Y, Hong Z, Chai Y (2019) Recent advances in microfluidic devices for bacteria and fungus research. TrAC Trends Anal. Chem 112: 175-195. https://doi.org/10.1016/J.TRAC.2018.12.024.

[3] McDonald JC WG, Duffy DC, Anderson JR, Chiu DT, Wu H, Schueller OJ (2000) Fabrication of microfluidic systems in poly(dimethylsiloxane). Electrophoresis 21: 27-40. https://doi.org/10.1002/(sici)1522-2683(20000101) 21:1\%3C27::aid-elps27\%3E3.0.co;2-c

[4] Fiorini GS, Chiu DT (2005) Disposable microfluidic devices: Fabrication, function, and application. Biotechniques 38: 429-446.https://doi.org/10. 2144/05383RV02.

[5] Whitesides GM (2006) The origins and the future of microfluidics. Nature 442: 368-373. https://doi.org/10.1038/nature05058.

[6] Convery N, Gadegaard N (2019) 30 Years of Microfluidic. Micro Nano Eng. 2: 76-91. https://doi.org/10.1016/j.mne.2019.01.003.

[7] Gale BK, Jafek AR, Lambert CJ, Goenner BL, Moghimifam H, Nze UC, Kamarapu SK (2018) A review of current methods in microfluidic device fabrication and future commercialization prospects. Inventions 3. https: //doi.org/10.3390/inventions3030060.

[8] Mashaghi S, Abbaspourrad A, Weitz DA, van Oijen AM (2016) Droplet microfluidics: A tool for biology, chemistry and nanotechnology. TrAC Trends Anal. Chem. 82: 118-125. https://doi.org/10.1016/j.trac.2016.05. 019 .

[9] Minzioni P, Osellame R, Sada C, Zhao S, Omenetto FG, Gylfason KB, Haraldsson T, Zhang Y, Ozcan A, Wax A (2017) Roadmap for opto fluidics. J. Opt. 19: 093003. https://doi.org/10.1088/2040-8986/aa783b

[10] Schembri F, Bucolo M (2011) Periodic input flows tuning nonlinear twophase dynamics in a snake microchannel. Nanofluidics 11 : 189-197. https: //doi.org/10.1007/s10404-011-0786-5.

[11] Harrison DJ, Fluri K, Seiler K, Fan Z, Effenhauser CS, Manz A (1993) Micromachining a Miniaturized Capillary Electrophoresis-Based Chemical Analysis System on a Chip. Science 261: 895-897. https://doi.org/10.1126/ SCIENCE.261.5123.895. 
[12] Jacobson SC, Hergenroeder R, Koutny LB, Ramsey JM (2002) Open Channel Electrochromatography on a Microchip. Anal. Chem. 66: 23692373. https://doi.org/10.1021/AC00086A024.

[13] Jacobson SC, Moore AW , Ramsey JM (2002) Fused Quartz Substrates for Microchip Electrophoresis. Anal. Chem. 67: 2059-2063. https://doi.org/ 10.1021/AC00109A026.

[14] Matzke CM, Kottenstette RJ, Casalnuovo SA, Frye-Mason GC, Hudson ML, Sasaki DY, Manginell RP, Wong CC (1998) Microfabricated silicon gas chromatographic microchannels: fabrication and performance. Photonics West - Micro and Nano Fabricated Electromechanical and Optical Components. https://doi.org/10.1117/12.324309.

[15] Moore AW, Jacobson SC, Ramsey JM (1995) Microchip Separations of Neutral Species via Micellar Electrokinetic Capillary Chromatography. Anal. Chem. 67: 4184-4189. https://doi.org/10.1021/ac00118a023.

[16] Becker C, Gartner H (2000) Polymer microfabrication methods for microfluidic analytical applications. Electrophoresis 21: 1226. https://doi.org/10.1002/(SICI)1522-2683(20000101)21:1\%3C12:: AID-ELPS12\%3E3.0.CO;2-7

[17] Chao TC, Ros A (2008) Microfluidic single-cell analysis of intracellular compounds. J.R. Soc. Interface 5: 139-150. https://doi.org/10.1098/RSIF. 2008.0233.FOCUS.

[18] Eriksson E, Sott K, Lundqvist F, Sveningsson M, Scrimgeour J, Hanstorp D, Goksor M, Graneĺi A (2010) A microfluidic device for reversible environmental changes around single cells using optical tweezers for cell selection and positioning. Lab Chip. 10: 617-625. https://doi.org/10.1039/ B913587A.

[19] Zheng B, Spencer L, Ismagilov RF (2003) Screening of protein crystallization conditions on a microfluidic chip using nanoliter-size droplets. J. Am. Chem. Soc. 125: 11170-11171. https://doi.org/10.1021/JA037166V.

[20] Chiu DT, Jeon NL, Huang S, Kane RS, Wargo CJ, Choi IS, Ingber DE, Whitesides GM (2000) Screening of protein crystallization conditions on a microfluidic chip using nanoliter-size droplets. Proc. Natl. Acad. Sci. 97: 2408-2413. https://doi.org/10.1073/PNAS.040562297.

[21] Benedetto A, Accetta G, Fujita Y, Charras G (2014) Spatiotemporal control of gene expression using microfluidics. Lab Chip. 14: 1336-1347. https://doi.org/10.1039/c3lc51281a. 
[22] Huber DL, Manginell RP, Samara MA, Kim BI, Bunker BC (2003) Programmed Adsorption and Release of Proteins in a Microfluidic Device, Science 301: 1336-1347. https://doi.org/10.1126/SCIENCE.1080759.

[23] Stroock AD, Whitesides GM (2003) Controlling flows in microchannels with patterned surface charge and topography. Acc. Chem. Res. 36: 597604. https://doi.org/10.1021/ar0202870.

[24] Xia Y, Whitesides GM (1998) Soft lithography, Angew. Chemie - Int. 37: 550-575. https://doi.org/10.1002/(sici)1521-3773(19980316)37:5/550:: aid-anie550 $>3.3 . c 0 ; 2-7$.

[25] Han D, Lee H (2020) Recent advances in multi-material additive manufacturing: methods and applications, Curr. Opin. Chem. Eng. 28: 158-166. https://doi.org/10.1016/J.COCHE.2020.03.004.

[26] Han D, Yang C, Fang NX, Lee H (2019) Rapid multi-material 3D printing with projection micro-stereolithography using dynamic fluidic control, Addit. Manuf. 27: 606-615. https://doi.org/10.1016/j.addma.2019.03.031.

[27] Cairone F, Davi S, Stella G, Guarino F, Recca G, Cicala G, Bucolo M (2020) 3D-Printed micro-optofluidic device for chemical fluids and cells detection, Biomed. Microdevices. 22: 1-10. https://doi.org/10.1007/ s10544-020-00487-3.

[28] Stella G, Skariya N, Gagliano S, Cicala G, Bucolo M (2021) Microoptofluidic systems for slug detection, MED'21 Puglia (Italy) 2021.

[29] Shrivastava A (2018) Introduction to Plastics Engineering, Introd. to Plast. Eng. i: 1-16. https://doi.org/10.1016/b978-0-323-39500-7.00001-0.

[30] Sendner C, Horinek D, Bocquet L, Netz RR (2009) Interfacial Water at Hydrophobic and Hydrophilic Surfaces: Slip, Viscosity, and Diffusion, Langmuir. 25: 10768-10781. https://doi.org/10.1021/LA901314B.

[31] Choi CH, Westin KJA, Breuer KS (2008) To Slip or Not to Slip: Water Flows in Hydrophilic and Hydrophobic Microchannels, ASME Int. Mech. Eng. Congr. Expo. Proc.: 557-564. https://doi.org/10.1115/ IMECE2002-33707.

[32] Zhou G, Yao SC (2011) Effect of surface roughness on laminar liquid flow in micro-channels, Appl. Therm. Eng. 31: 228-234. https://doi.org/ 10.1016/j.applthermaleng.2010.09.002.

[33] Alford TL, Feldman LC, Mayer JW (2007) Fundamentals of Nanoscale Film Analysis, Springer Science Business Media. 
[34] Weilin Q, Mala GM, Dongqing L (2000) Pressure-driven water flows in trapezoidal silicon microchannels, Int. J. Heat Mass Transf. 43: 353-364. https://doi.org/10.1016/S0017-9310(99)00148-9.

[35] Pfund D, Rector D, Shekarriz A, Popescu A, Welty J (2000) Pressure drop measurements in a microchannel, AIChE J. 46: 1496-1507. https: //doi.org/10.1002/AIC.690460803.

[36] Warr CA, Hinnen HS, Avery S, Cate RJ, Nordin GP, Pitt WG (2021) 3D-Printed Microfluidic Droplet Generator With Hydrophilic and Hydrophobic Polymers, Micromachines. 12: 1-13. https://doi.org/10.3390/ mi12010091.

[37] Eduati F, Utharala R, Madhavan D, Neumann UP, Longerich T, Cramer T, Saez-Rodriguez J, Merten CA (2018) A microfluidics platform for combinatorial drug screening on cancer biopsies, Nat. Commun. 9. https: //doi.org/10.1038/s41467-018-04919-w.

[38] Song R, Abbasi MS, Lee J (2019) Fabrication of 3D printed modular microfluidic system for generating and manipulating complex emulsion droplets, Microfluid. Nanofluidics. 23: 1-11. https://doi.org/10.1007/ s10404-019-2258-2.

[39] Sun YS (2016) Comparison of chip inlet geometry in microfluidic devices for cell studies, Molecules. 21. https://doi.org/10.3390/molecules21060778. 